\title{
Overview of Aqua MODIS 10-year On-orbit Calibration and Performance
}

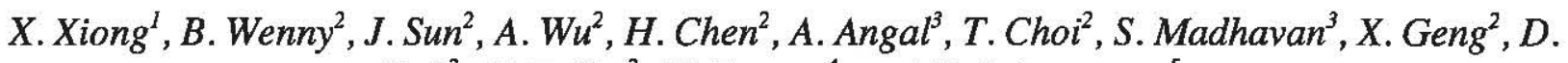 \\ Link $^{2}$, G. Toller ${ }^{2}$, W. Barnes ${ }^{4}$, and V.Salomonson ${ }^{5}$, \\ ${ }^{1}$ Sciences and Exploration Directorate, NASA/GSFC, Greenbelt, MD 20771, USA \\ ${ }^{2}$ Sigma Space Co., 4801 Forbes Boulevard, Lanham, MD 20706, USA \\ ${ }^{3}$ Sciences and System Applications Inc., 10210 Greenbelt Road, Lanham, MD 20706, USA \\ ${ }^{4}$ University of Maryland, Baltimore County, 1000 Hilltop Circle, Baltimore, MD 21250, USA \\ ${ }^{5}$ University of Utah, Salt Lake City, UT 84112, USA
}

Since launch in May 2002, Aqua MODIS has successfully operated for nearly 10 years, continuously collecting global datasets for scientific studies of key parameters of the earth's land, ocean, and atmospheric properties and their changes over time. The quality of these geophysical parameters relies on the input quality of sensor calibrated radiances. MODIS observations are made in 36 spectral bands with wavelengths ranging from visible (VIS) to longwave infrared (LWIR). Its reflective solar bands (RSB) are calibrated using data collected from its on-board solar diffuser and regularly scheduled lunar views. The thermal emissive bands (TEB) are calibrated using an on-board blackbody (BB). The changes in the sensor's spectral and spatial characteristics are monitored by an on-board spectroradiometric calibration assembly (SRCA). This paper presents an overview of Aqua MODIS 10-year on-orbit operation and calibration activities, from launch to present, and summarizes its on-orbit radiometric, spectral, and spatial calibration and characterization performance. In addition, it will illustrate and discuss on-orbit changes in sensor characteristics and corrections applied to continuously maintain the sensor level 1B (L1B) data quality, as well as lessons learned that could benefit future calibration efforts. 